\title{
Pharmacovigilance Considerations for Biosimilars in the USA
}

\author{
Gustavo Grampp $^{1}$ Thomas Felix ${ }^{2}$
}

Published online: 29 September 2015

(c) The Author(s) 2015. This article is published with open access at Springerlink.com

\begin{abstract}
In 2015, five or more biosimilars may be approved in the USA. Because no two biologic medicines are identical, postapproval safety monitoring will be critical to detect potential differences in safety signals between a biosimilar, its reference product, and other biosimilars. Postapproval safety monitoring in the USA uses two signal detection systems: spontaneous reporting systems (SRSs) and active surveillance (AS) systems. Both depend on accurate identification of the specific product(s) dispensed or administered to patients, which may be compromised when products from multiple manufacturers share common drug nomenclature or coding. Product identification can present challenges across different healthcare settings, including inpatient and ambulatory care. Common oraldosage drugs are predominantly dispensed directly to patients by pharmacists, whereas most injectable drugs, including biologics, are administered to patients by healthcare professionals in outpatient clinics or hospitals. Thus, the effectiveness of SRS and AS mechanisms in both pharmacy and medical channels must be given greater consideration as biotechnology matures. In this article, we describe these systems and their limitations. We identify challenges and opportunities for product-specific safety
\end{abstract}

Thomas Felix

thfelix@amgen.com

Gustavo Grampp

ginog@amgen.com

1 Amgen Inc., Longmont, CO, USA

2 Amgen Inc., 601 13th Street NW, Washington, DC 20005, USA surveillance of biologics in both the pharmacy and medical settings and provide recommendations to improve biologic safety surveillance under the current and future systems envisioned in the Drug Quality and Security Act. As biosimilars are integrated into existing pharmacovigilance systems, distinguishable nonproprietary names and codes for all biologics, as well as other opportunities to improve traceability (e.g., increased use of barcodes), must be considered to ensure patient safety and confidence in this new class of drugs.

\section{Key Points}

Postapproval safety monitoring for biologic products relies on both active surveillance (AS) and spontaneous reporting systems (SRSs), and these must be effective for biologics dispensed both in the pharmacy and through medical benefit channels.

Both SRS and AS approaches rely on accurate identification of the product(s) dispensed or administered to patients, and the effectiveness of these surveillance methods may be compromised when there are multiple manufacturers for products that share common drug nomenclature or coding.

Federal, state, and health information technology policies that promote complete, accurate, and accessible tracking of dispensing data in patient medical records are essential to ensure traceability of outcomes for biologic products. These should include, but not be limited to, the application of distinguishable product nomenclature and reimbursement coding. 


\section{Introduction}

2015 marks an important milestone in the maturity of medical biotechnology, with five or more biosimilar applications pending review by the US Food and Drug Administration (FDA). For the first time, a number of manufacturers will produce a series of highly similar but not identical medicines for the US market. The first biosimilar (Zarxio ${ }^{\circledR}$ [filgrastim-sndz]) was approved in the USA in March 2015, indicating that this new era has begun [1]. Accordingly, it will be important that pharmacovigilance systems are able to detect differences between adverse events (AEs) associated with a biosimilar and AEs associated with its reference product [2-4]. Given the structural and manufacturing complexities of biologics and the potential for structural differences between biosimilars and their reference products, the paradigm currently applied for monitoring of drug safety with small-molecule generic drugs is insufficient for biologics, including biosimilars [5]. Long-term patient safety monitoring of biologics is required to properly evaluate the immunogenic effects of both new and established biologics in the market [6]. Postapproval surveillance for immunogenicity and rare AEs may be needed; they may not be apparent during preapproval testing, because of the relatively small patient populations evaluated [2]. Moreover, it is important that the specific biologic or manufacturer is readily identified to ensure accurate tracing of AEs to the administered product [7]. Increased use of barcodes on biologic drugs should improve tracing capabilities, as should implementation of the US Drug Quality and Security Act/Drug Supply Chain Security Act (DQSA/DSCSA), which outlines use of an interoperable electronic system to identify and trace prescription drugs in the USA [8].

The objectives of this article are (1) to describe the spontaneous reporting systems (SRSs) and active surveillance (AS) systems used in the USA and their limitations; (2) to inform regulators, physicians, and pharmacists about the pharmacovigilance challenges for biologics from multiple manufacturers that have similar active substances; (3) to describe the difficulties of product traceability in hospital and physician office settings - the predominant settings for the administration of biologics; (4) to describe challenges and opportunities to improve product-specific safety surveillance of biologics dispensed to patients in retail or mail-order pharmacy settings; and (5) to encourage improvements in the traceability and pharmacovigilance of biologics in the USA by making recommendations for policies and practices that may improve the fidelity of SRSs and AS systems, targeting those recommendations toward the settings where these pharmacovigilance systems are used.

\section{USA Pharmacovigilance and Biologics}

In the USA, postapproval safety signal detection is performed primarily using SRS and AS systems [9, 10]. The attributes of each of these systems can be complementary for biologics from multiple manufacturers that have similar active substances, provided that AEs are properly linked to a specific product.

SRSs (e.g., MedWatch and institution-based reporting) are considered passive surveillance methods, which rely on voluntary reports from physicians, pharmacists, other healthcare providers, and patients [11]. AS methods include retrospective analysis of medical records at Sentinel-affiliated sites and drug or disease registries, as well as use of drug event monitoring (e.g., surveys of patients identified through electronic prescription data) [12]. SRSs are important for identification of safety signals, including potential rare AEs not identified during clinical trials or premarketing studies [10]. For products (such as biologics) that are relatively sensitive to manufacturing conditions, SRSs may be useful for detection of emergent safety signals associated with changes in product quality throughout the life cycle of the medicine. A limitation of SRS approaches is that they cannot accurately quantify the incidence of identified risks for a given product, because the total number of patients treated with the drug is unknown $[10,13]$. AS methods can identify new safety signals [14] but are better suited to assess the incidence and severity of identified risks [10]. AS systems, when used as a method to identify new safety signals, often derive multiple potential links, necessitating development of algorithms that are informed by and integrated with clinical and scientific assessment to further prove causality; the SRS and other sources may be used to prespecify potential AEs of interest [15].

Both SRS and AS approaches rely on accurate identification of the product(s) dispensed or administered to patients, and the effectiveness of these surveillance methods may be compromised when there are multiple manufacturers of products that share common drug nomenclature or coding [4]. Furthermore, product identification can present unique challenges across different healthcare settings, including inpatient and ambulatory care. Common oral-dosage drugs are predominantly dispensed directly to patients by pharmacists (i.e., via the pharmacy benefit channel), whereas most injectable drugs, including biologics, are administered to patients by healthcare professionals in outpatient clinics or hospitals, as covered under the medical benefit. Thus, it is essential to account for the effectiveness of SRS and AS mechanisms in both the pharmacy and medical channels. 


\subsection{USA MedWatch Spontaneous Reporting System}

Spontaneous surveillance occurs through reports to the manufacturer and to the FDA MedWatch program. Physicians, pharmacists, other healthcare providers, and patients can voluntarily submit reports of serious reactions, product quality problems, and therapeutic failure, through a MedWatch reporting form [11]. Indeed, according to a published analysis of suspected drug reaction reports on biopharmaceutical products in the FDA Adverse Event Reporting System (FAERS) database (from 2004 to 2010), patients were identified as the reporter in $41 \%$ of reports, prescribers or other healthcare providers in $36 \%$ of reports, and pharmacists in only $3 \%$ of reports [16]. SRSs rely on the accuracy of the information submitted, and, on the basis of the distribution of likely reporters of AEs, it is not sufficient that the pharmacist is the only member of the care team who has ready access to complete records identifying the drugs administered.

Under the FDA's current system, MedWatch reports can be submitted with minimal product identification. In brief, these reports include the following data: an identifiable patient, an identifiable reporter, a suspect drug or biologic product, and an $\mathrm{AE}$ or fatal outcome [17]. Reports may contain only a nonproprietary active ingredient name; therefore, drugs with the same nonproprietary name will typically be grouped together for pharmacovigilance purposes [18]. The FDA's manual of policies and procedures titled Handling of Adverse Experience Reports and Other Generic Drug Postmarketing Reports stipulates that "generally, OGD [the Office of Generic Drugs] receives few AERs [adverse experience reports] or similar reports since the reports may not specify a generic manufacturer for the drug product. Furthermore, the safety profile of a particular drug is usually well-known before generic versions are approved. Therefore, AERs associated with a generic drug are less likely to be reported" [18]. Although the MedWatch form advises that the manufacturer name should be reported when the nonproprietary name is used, the FDA has indicated that the manufacturer name is included in only $3 \%$ of reports, and the associated data field was therefore removed from the FAERS database in 2000 [19].

A further complication of the pharmacovigilance of biologics is that for delayed immunologic reactions-in particular, those caused by formation of anti-drug antibodies-there may be a considerable time lag between administration of the biologic and the appearance of a serious reaction [20]. This time lag may make it difficult to attribute the event to a specific product in a case in which a patient has been switched between related biologics. A delayed immune response may also affect the ability to attribute the $\mathrm{AE}$ to a specific lot of a biologic product if a comprehensive medication history is not accessible $[3,21]$. In these circumstances, the SRS would be inadequate for identifying manufacturer- or batch-associated issues with biologics. However, with modifications as described below, SRS AE reports could also be a means of achieving early identification of batch- or product-specific problems. In a pharmacovigilance environment, with multiple manufacturers selling products with similar active substances, it is critical that product identification is supported by robust and, if possible, redundant product-specific identifiers. There are two case studies that may have direct implications for AE reporting in SRSs after biosimilars are introduced in the USA [22, 23].

In the first case study, an analysis of the FAERS demonstrated that for six of the eight drugs evaluated, the number of monthly AE reports attributed to the originator brand product did not decrease significantly when generic competition became available, although there was a steep decrease in the number of dispensed prescriptions of the originator drug [22]. This suggests that AEs may have been attributed to the wrong product through use of the originator brand name in SRS reports, when it is possible that the patients received a generic product [22].

The limitations of the existing US SRSs that can compromise an efficient and expeditious investigation are exemplified in a second case study of 246 death reports potentially associated with contaminated heparin administration between January 2007 and May 2008 [24]. The US Government Accountability Office report on the FDA's management of the heparin contamination crisis indicated that, on review of 94 death reports, only 13 of the reports included heparin lot numbers, and 28 of the 46 voluntary reports did not identify the heparin manufacturer. Therefore, it was not feasible for the FDA to determine the heparin contamination status in most of the deaths [23]. Economically incentivized adulteration of raw materials is not a common concern for biologics, but this case study highlights current gaps in SRSs that prevent accurate and timely identification of an implicated product and manufacturer when related products from multiple manufacturers are available to patients.

\subsection{USA FDA's Sentinel System and Other Sources of Data (Active Surveillance)}

AS methods are diverse, and a complete characterization of this comprehensive area is beyond the scope of this article. As such, our discussion is limited to retrospective database analyses from existing systems and does not include more expensive methods (e.g., registries, open-label long-term studies, prospective observational studies). Retrospective AS occurs through analysis of larger patient administrative 
data sets from healthcare institutions, laboratories, individual healthcare providers, and/or medical claims records [25]. As one example of AS, the FDA's Sentinel pilot program uses the electronic health information available in claims systems, inpatient and outpatient healthcare records, and patient registries to match a specific medicine (assuming billing codes are product specific) with a clinically reported outcome [26]. In principle, billing and electronic health record (EHR) databases can refer to reimbursement codes to faithfully record claims associated with use of a specific drug, as well as diagnosis codes linked to potential AEs, permitting statistical linkage of therapies to patient outcomes. Vulnerabilities include both the accuracy of product identification as derived from claims data and, if captured, the diagnosis coding selected by the clinician. Pooled administrative and claims data often shed product identifiers such as brand names when merged from disparate systems, leaving only nonproprietary names or active ingredient names connected to a patient's medical record. Regardless of the data source (e.g., claims, general clinical practice, drug dispensary databases), productspecific AS requires either specific reimbursement codes or use of unique product names or other identifiers (e.g., manufacturer names).

AS using medical claims data is only sensitive to billable International Classification of Diseases, Ninth Edition (ICD-9) diagnosis codes and diagnosis-related groups (DRGs) used to link administration of a drug or biologic to a subsequent AE. A systematic review of the Mini-Sentinel program found limited information related to anaphylaxisreporting algorithms and indicated that the positive predictive value of ICD-9 codes for anaphylaxis was low when applied to all-cause anaphylaxis [27]. There are many nonspecific ICD-9 codes that have been used when categorizing immunologic reactions, including (but not limited to) allergic urticaria (708.0), unspecified adverse effect of a drug (995.2), allergy unspecified (995.3), and erythema multiforme (695.1) [28-30]. Because many ICD-9 codes are currently used to capture allergic and other immunologic reactions, it is likely that this would also be true for biologics, including immunologic reactions leading to a loss of efficacy.

In a pharmacovigilance setting for biologic products subject to biosimilar competition, an opportunity to enable accurate attribution of an $\mathrm{AE}$ to a specific product may include assignment of multiple or redundant product identifiers to promote resilience of attribution in the event of errors or ambiguity in any one identifier. The relative effectiveness and utility of these product identifiers is dependent on the settings in which the product is predominantly used and the ability of SRSs and AS systems to accurately capture the product identity.

\section{Utility and Limitations of Product Identifiers for Biologics in Spontaneous Reporting and Active Surveillance Systems}

The five product identifiers that may be included in MedWatch $\mathrm{AE}$ reports are the brand name, nonproprietary name, National Drug Code (NDC), manufacturer name, and lot number [3]. Other product identifiers, such as medical benefit reimbursement codes (Healthcare Common Procedure Coding System [HCPCS]), may be used for AS. The utility of each of these identifiers may be limited in regard to their use in SRSs and limited by the healthcare setting in which the $\mathrm{AE}$ reporting is performed (see Table 1).

\subsection{Brand Names}

Brand names are often the primary identifiers used for reporting AEs [3]. Approximately $50 \%$ of 376 USA prescribers who were surveyed used the brand name to identify a medicine for prescription or recording in patient records [31]. Brand name reporting for biologics in SRSs can vary by the product class and jurisdiction. For example, $84 \%$ use of accurate brand names has been reported for insulins in the USA, whereas product-specific attribution of epoetins approached $99 \%$ in the European Union (EU) $[16,32]$. In the EU, legislation enacted in 2010 requires member states to take measures to ensure that trade names are used in health records and AE reports for biological medicinal products [33]. Despite this legislation, $21 \%$ of filgrastim-related spontaneous reports originating from the EU between April 2012 and December 2014 and received by Amgen were not attributed to a particular brand name. Similarly, in Australia, $42 \%$ of reports received by the Therapeutic Goods Administration public database between March 2011 and November 2014 were coded as "filgrastim (not specified)" [34]. These data indicate that a large proportion of reporters use the nonproprietary name, not the brand name, when reporting AEs. Brand names are not usually found in medical benefit claims and are unsuitable for AS, in which only billing codes are assigned to administered products.

\subsection{Nonproprietary Names}

A product's nonproprietary name (also referred to as its generic name, established name, US Adopted Name [USAN], International Nonproprietary Name [INN], or active ingredient) is often used in the USA because it is not mandatory that products receive a brand name, nor is it mandatory that prescribers use a brand name when one is available. A survey of community-based physician office 


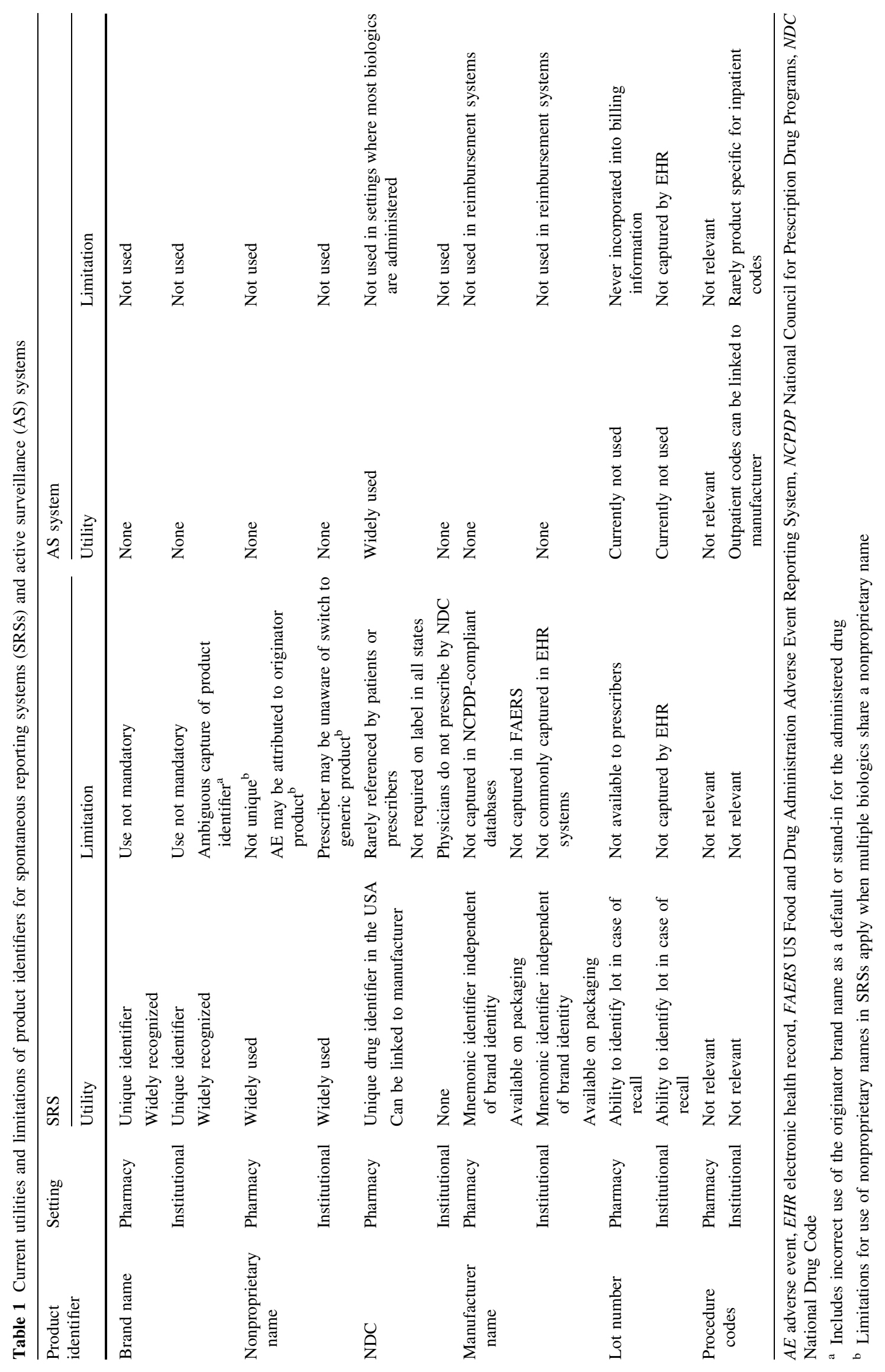


visits in the USA revealed that the median frequency of generic name prescribing was $21 \%$ [35]. The nonproprietary name is commonly used to identify the product in AE reports and is acknowledged as a useful tool for pharmacovigilance of nonbiologic drugs worldwide [3, 6].

Most nonproprietary names for drugs and biologics are linked to the World Health Organization (WHO) INN system. In many cases, INN naming rules would permit sponsors to differentiate between originator biologics and their copies through incorporation of distinct Greek letter suffixes (e.g., "epoetin zeta" versus "epoetin alfa") when differences in glycosylation exist. However, such a system is voluntary, and, in practice, the INN cannot be relied on for product-specific identification of biosimilars, because regulators have generally permitted biosimilars to adopt the same INN as their reference products $[6,16]$. If multiple biosimilars were licensed with a shared nonproprietary name identical to the INN of the same reference product, patients and physicians might be unable to distinguish between the biologics for the purposes of AE reports. To address this risk, the FDA has recently published draft guidance that calls for the addition of a unique suffix to the INN for all biologics, including biosimilars, to differentiate products and minimize inadvertent substitution [36]. This naming convention is similar to that proposed by the WHO [37]. The success of a naming approach that confers unique, distinguishable names for all biologics will be limited by its implementation and follow-through. If unique names are confirmed for all biosimilars, then the naming approach will need to be accommodated and used consistently in all settings and systems in which biologics are prescribed, dispensed, recorded, and, if needed, reported for AEs.

\subsection{National Drug Codes}

NDCs are specific to each drug product but are rarely used by physicians. For example, only $14 \%$ of respondents to a survey of prescribers conducted by the Alliance for Safe Biologic Medicines indicated that they would use the NDC or a combination of the product name and the NDC in an AE report [31]. Moreover, NDCs are not required by federal law to appear on product labeling [38], and they are rarely found on the labels of dispensed prescription medicines [39]. Thus, NDCs have limited use as reliable product identifiers to support the SRS. However, NDCs are commonly used for reimbursement in the community pharmacy setting and can provide accurate product identification for pharmacy benefit claims databases used in AS. Finally, NDCs are used only in the USA and are not a global solution for product identification.

\subsection{Manufacturer Names}

A field for the manufacturer name is included in the MedWatch 3500 report form as an optional identifier for unbranded products [40], but current pharmacovigilance systems in the USA are not set up to capture this identifier for nonvaccine biologic products. The MedWatch form advises reporters to include the manufacturer name when using a generic drug name [39, 40], but the field is used so rarely that the FDA does not include the manufacturer name in the FAERS database [19]. In the USA, the Vaccine Adverse Event Reporting System (VAERS), sponsored by the Centers for Disease Control and Prevention and the FDA, separately tracks AEs that occur after vaccine administration; the manufacturer name is commonly tracked in VAERS [41, 42]. EHR systems may not include a field for the manufacturer name, nor is it included in the National Council for Prescription Drug Programs (NCPDP) 10.6 Script standard for medication history reports [43]. Therefore, significant changes in database structures, regulations, policies, and incentives would need to be implemented to increase the use of manufacturer names for nonvaccine biologic products in health records and safety surveillance systems.

\subsection{Lot Numbers}

Lot numbers are of primary importance in assisting manufacturers with connection of a safety issue to a specific lot of a manufactured product. The lot number is the only product identifier that can provide information for tracing of specific manufacturing production batches [3]. Billing data collected in hospital and physician office settings typically do not include lot numbers [44]; therefore, lot numbers have no utility with respect to AS. Additionally, these data are not routinely used in the SRS; a review of the completeness of MedWatch data indicated that lot numbers were completed in only $9 \%$ of the 10.2 million records evaluated [45]. In a review of reports from FAERS and EudraVigilance in the EU, lot numbers for biopharmaceuticals were provided in 24 and $21 \%$ of reports, respectively [16]. Batch number reporting depends on the product class. An analysis of FAERS suspect AE reports for insulin and somatropin products showed that lot numbers were available in $37 \%$ of insulin reports but in only $13 \%$ of somatropin reports [32].

In light of the limitations of each of these five product identifiers, there is a need for reliable and redundant identifiers for biologic products subject to biosimilar competition, especially in AS systems that are designed to capture data through analysis of health records. 


\subsection{Medical Channel Reimbursement Codes}

Physician office and hospital outpatient claims for drug administration procedures commonly use HCPCS codes, but these codes have not historically been assigned uniquely for each new biologic [46]. Because this channel represents the majority of therapeutic biologic product prescriptions in the USA, such codes are particularly relevant to AS of biologics. A recent study confirmed that claims-based data could be used to track immunogenicity-related signals for an injectable generic in the pharmacy setting but not for hospital or clinic use, where all versions of the product share the same HCPCS codes [47].

However, on July 8, 2015, the Centers for Medicare \& Medicaid Services (CMS) published in the Federal Register a proposed rule to have all biosimilars share a J-code [48]. Such a policy would prevent AS of biosimilar products, allowing comparisons only between the experience with a reference product and the aggregate experience of its biosimilars. This proposed rule goes against the legislation establishing the biosimilar pathway in the USA, which was supported by conforming amendments to the Social Security Act section 1847A(b)(4),(6) that should have prohibited reimbursement based on a volumeweighted average $[49,50]$ and supported separate HCPCS codes for each biosimilar product. If the proposed rule is reversed and unique HCPCS codes are issued for biosimilars, it may be possible to perform product-specific AS.

A small proportion of biologic therapies may be administered in the hospital inpatient setting. Hospital inpatient claims typically use a DRG to assign a bundled payment according to the ICD-9 Clinical Modification diagnosis and procedure codes [46]. With very rare exceptions, these procedure codes are not specific to a given biologic therapy or underlying event; therefore, they are not useful for AS.

\section{Robust Pharmacovigilance of Biologics in the USA Must Account for the Settings of Use}

To more completely evaluate and understand the current capabilities and limitations of pharmacovigilance systems for biologics with multiple manufacturers, it is necessary to consider all of the settings where therapeutic biologics are used. The majority of therapeutic biologic products are administered by healthcare professionals in physician offices, hospital outpatient settings, or other institutional settings, but some biologics are distributed primarily through retail or mail-order pharmacy channels (e.g., selfadministered therapeutics for chronic diseases such as rheumatoid arthritis) [44].

\subsection{The Outpatient Medical Channel}

In the absence of a distinguishable nonproprietary name and product-specific billing codes, health records may not include a unique verifiable product identifier associated with a biologic administered in physician offices and hospital outpatient settings. The lack of distinguishable nonproprietary names and product-specific billing codes could undermine the effectiveness of both SRS and AS systems. If an office or hospital pharmacy has the option to purchase similar biologics from multiple sources that are assumed to be the same because of shared nonproprietary names, there may be a lack of transparency for the prescriber with respect to the medicine purchased, dispensed, and administered to the patient. This would create confusion in the event that one biosimilar, but not others, was associated with a particular AE.

A depiction of how an $\mathrm{AE}$ caused by a generic product can be misattributed to the originator product is provided in Fig. 1. When a generic supply of medication is introduced into the in-house pharmacy inventory, administrative staff may not code new identifier records in the formulary that are specific to the generic. Instead, because the products share the same nonproprietary names, the generic may simply be identified in the prescription order entry and EHR systems as if the originator brand product were being ordered and dispensed. Failure of the EHR to include information about the manufacturer of the generic product actually dispensed, along with use of the originator brand name as an alias for the generic, may result in incorrect use of the originator brand name in $\mathrm{AE}$ reports.

\subsection{The Pharmacy Channel}

The pharmacy channel represents another potential challenge to pharmacovigilance, because dispensing transactions are typically performed by an entity independent of the prescriber, creating a potential gap in provider access to product identifiers. This challenge is particularly acute in the case of automatic substitution of products that are determined to be therapeutically equivalent. In the context of biologic medicines, many are anticipated to be approved without a determination of interchangeability, whereas some biosimilars may ultimately be licensed as interchangeable biologics that may be automatically substituted for the prescribed reference biologic [51]. When an automatic substitution is deemed scientifically appropriate, the provider's prescription records will be rendered either ambiguous or inaccurate, depending on the identity of the dispensed product. Some state pharmacy practice acts have incorporated amendments intended to address this potential gap. If automatic substitution of a biologic is permitted in a given state, these provisions promote physician access to medication history data by requiring that pharmacists enter 


\section{Medication Order Administration Process}

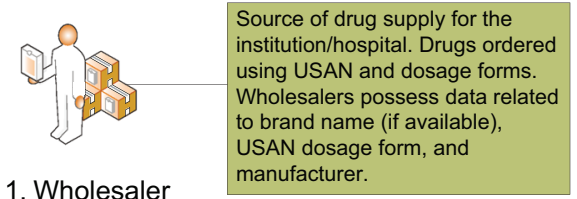

1. Wholesaler

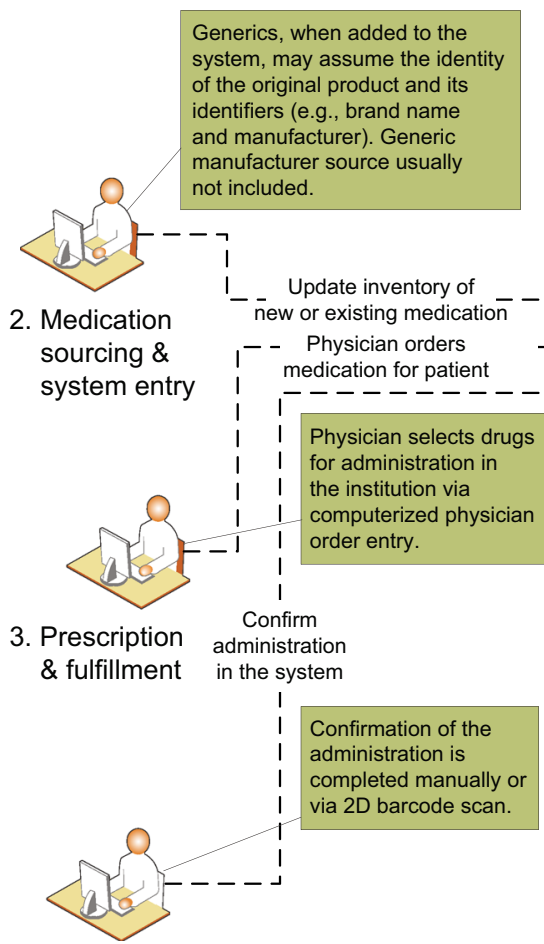

4. Administration

Fig. 1 Schematic of prescription order entry and fulfillment at a US medical institution, demonstrating how reported adverse events (AEs) can be misattributed. When a generic supply of medication is introduced into the in-house pharmacy inventory, administrative staff may not introduce new identifier records specific to the generic.

product identifiers into an electronic record system accessible to prescribers. In some states, the amended practice acts may require direct communication of the dispensing data to the prescriber by other means if such interoperable systems are not in place. For example, a recently passed Delaware Senate bill includes a requirement for the pharmacist to record the name and manufacturer of a biologic product within 10 days of dispensing the product when an FDA-approved interchangeable biologic product is substituted for a prescribed reference biologic product. The pharmacist must also, within 10 days, inform the prescribing physician which biologic was dispensed [52]. A similar bill was passed in Massachusetts, requiring pharmacists to record substitution of interchangeable biologic products in an interoperable EHR system, if available, and to report such substitutions to the prescriber [53].

Finally, AS of biologics used in the pharmacy setting is facilitated by use of product-specific reimbursement codes
AE Reporting Process

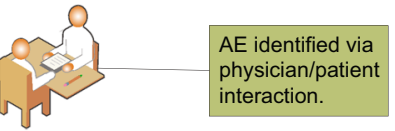

1. AE identified

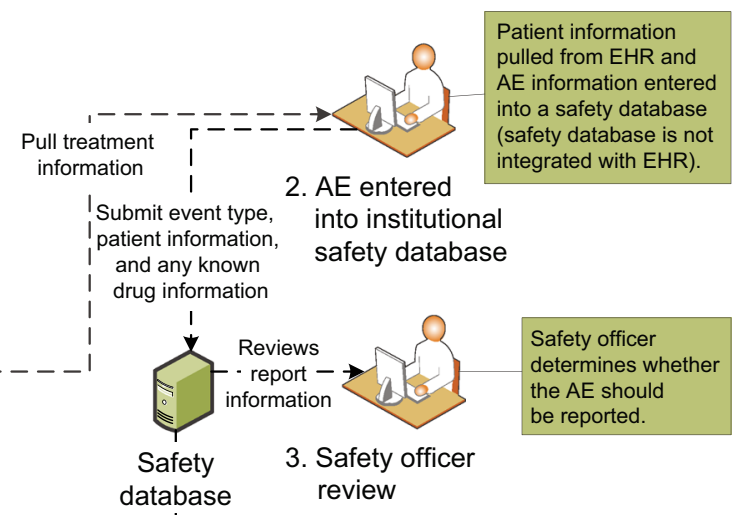
review
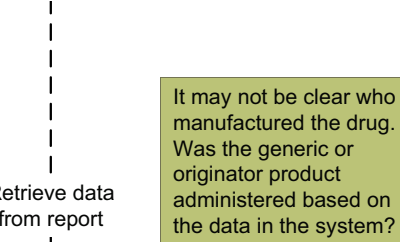

the data in the system?

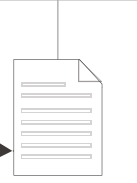

4. Complete MedWatch 3500 report
5. MedWatch report filed with the FDA
Instead, the generic may be identified in the prescription order entry and electronic health record (EHR) systems as if the originator product were being ordered and dispensed. $2 D$ two-dimensional, FDA US Food and Drug Administration, USAN US Adopted Name

(NDCs). This would also be the case if interchangeable biologics are automatically substituted. Reimbursement codes are available in the administrative claims databases and may be linked to AE-related diagnosis codes irrespective of provider awareness of the specific biologic dispensed.

\section{Four Dimensions to Consider for Effective Biologic Pharmacovigilance: Limitations and Recommendations for Accurate Product Identification}

The scope of the challenges to improving the robustness of SRS and AS systems for biologic products subject to biosimilar competition is broad and encompasses both pharmacy and institutional settings (Fig. 2). Within each of the dimensions (i.e., combinations of pharmacovigilance 


\section{Pharmacy}

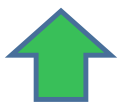

What works

- Pharmacist adverse event reporting

- Brand name reporting

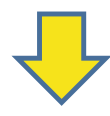

Current limitations

- Prescriber records with substitution

- Lot numbers not available to prescribers

- Generic name prescribing records

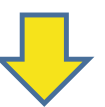

Current limitations

- Inconsistent coding of

immunologic reactions

- No lot numbers

\begin{tabular}{l}
\hline Policy recommendations \\
- Assignment of distinguishable nonproprietary names \\
- Amend standards to capture manufacturer name and lot numbers \\
in EHRs (including via DSCSA) \\
- MedWatch prompts for brand name, nonproprietary name, \\
manufacturer, and lot number \\
- Interoperable EHRs or pharmacist-prescriber communication \\
\hline
\end{tabular}

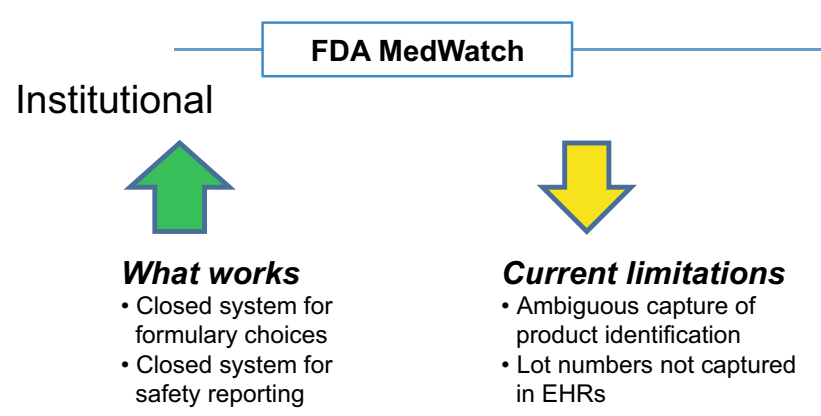

Policy recommendations

- Assignment of distinguishable nonproprietary names

- Amend standards to capture manufacturer name and lot numbers

in EHRs (including via DSCSA)

- MedWatch prompts for brand name, nonproprietary name,

manufacturer, and lot number

- Standards for improved EHR fidelity to products dispensed

Fig. 2 Four dimensions to consider for effective biologic pharmacovigilance. DSCSA Drug Supply Chain Security Act, EHR electronic health record, FDA US Food and Drug Administration, HCPCS Healthcare

mechanisms and settings of use), there are practices that currently allow for effective pharmacovigilance monitoring, as well as limitations that could hinder efforts to effectively monitor the safety of biologic products with multiple manufacturers.

We previously conducted a retrospective analysis to assess the capabilities of passive and active surveillance systems to track safety events for Lovenox ${ }^{\circledR}$ (enoxaparin; Sanofi) and generic enoxaparin-a complex, sterile injectable drug [47]. Enoxaparin was selected as a surrogate for biologics and biosimilars with respect to safety surveillance considerations because of its potential for immunogenicity and related rare safety signals (i.e., heparin-induced thrombocytopenia) and because enoxaparin is dispensed in both retail and non-retail channels. After the loss of exclusivity (LOE) for Lovenox, only $5 \%$ of spontaneous safety reports were processed by
Common Procedure Coding System, NDC National Drug Code, $R x$ prescription

Policy recommendations

- Product-specific HCPCS codes for biologics

- Improve diagnostic codes for immunologic reactions

- Assignment of distinguishable nonproprietary names

- Promote mandatory use of NDCs

- Amend standards to capture manufacturer name

and lot numbers in claims data

\section{Current limitations}

- Nonspecific product coding

- Inconsistent coding of

immunologic reactions

- No lot numbers

\section{Policy recommendations}

- Amend standards to capture manufacturer name

and lot numbers in claims data

\section{Sentinel Initiative}

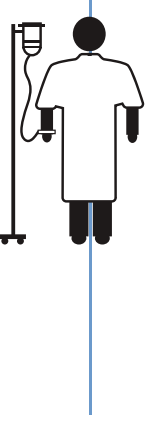

manufacturers of generic enoxaparin, although generic enoxaparin had rapidly captured $50 \%$ of the market after LOE. On the basis of the market share after LOE, reports attributable to specific generic enoxaparin products were roughly ninefold lower than expected. Insurance claims data supported useful AS of enoxaparin dispensed under a pharmacy benefit but not under a medical benefit. These results suggest that current safety surveillance systems do not sufficiently distinguish product-specific safety signals for drugs distributed by multiple manufacturers, including generics and biosimilars.

Because pharmacists have access to all required data on the drug dispensed, AE reporting via the SRS should work in the pharmacy setting, provided that other stakeholders (e.g., prescribers, other healthcare professionals, and patients) can also access these data. However, the effectiveness of the SRS in the pharmacy setting may be 
compromised because lot numbers are not available to the prescriber, and because prescriber records may not reflect the actual product dispensed in the case of a prescription by nonproprietary name or an automatic pharmacy substitution, thereby limiting accurate patient and prescriber $\mathrm{AE}$ reporting. In principle, medication history reports may provide prescribers with automated access to records of biologics dispensed in the pharmacy channel [54], but these reports will not facilitate the SRS unless specific product manufacturer identifiers are captured in those reports. To address these limitations, the following policy recommendations are suggested: (1) assignment of distinguishable nonproprietary names to all biologics; (2) amendment of standards to capture manufacturer names and lot numbers in EHRs (similar to the inclusion of NDCs in the NCPDP 10.6 Patient Medication History Script standard) [43]; (3) MedWatch online prompts for the product brand name (if available), nonproprietary name, manufacturer name, and lot number for biologics; and (4) establishment of interoperable EHRs or pharmacist-prescriber communication of dispensing transaction data that conveys productand manufacturer-specific information. In addition, education of the healthcare community on the importance, process, and best practices for reporting is also essential to improve the quality of SRS data. This may increase the number of rare or unexpected AEs reported while concurrently improving the quality of AE reports.

A closed system for formulary choices for products that are not assumed to be therapeutically equivalent and for safety reporting can, in principle, support the SRS in the institutional setting. The effectiveness of the SRS in the institutional setting is limited by ambiguous capture of product identification and the fact that lot numbers are not captured in EHRs. To address these limitations, the following policy recommendations are suggested: (1) assignment of distinguishable nonproprietary names to all biologics; (2) amendment of standards to capture manufacturer names and lot numbers in EHRs; (3) MedWatch online prompts for the product brand name (if available), nonproprietary name, manufacturer name, and lot number for biologics; and (4) promotion of standards for improved EHR fidelity with respect to the products dispensed.

In the AS pharmacy setting, NDCs are used widely, allowing for accurate product identification. However, ICD-9 diagnostic codes may not be clearly defined or consistently used for immunologic or allergic drug reactions (e.g., hypersensitivity) [55], and the lack of manufacturer identification and lot numbers in reimbursement claims data limits the effectiveness of AS for tracking issues related to changes in product quality. Improvement of diagnostic codes for immunologic reactions and policy changes to standardize the use of manufacturer identification and lot-level data within administrative data would increase the effectiveness of AS. This would include efforts to improve identification and reporting of potential immunogenicity-mediated loss of efficacy.

In the AS institutional setting, product-specific HCPCS codes are effective when they are available and are used. The effectiveness of AS in the institutional setting is limited when there is nonspecific product coding, inconsistent immunologic reaction diagnosis coding, and infrequent use of lot numbers. To address these limitations, establishment of product-specific HCPCS codes for biologics and improvement of diagnostic codes for immunologic reactions would increase the effectiveness of AS in the institutional settings in which biologic products are primarily administered [44]. Distinguishable nonproprietary names may also improve attribution in claims data linked to EHRs, even in the event that reimbursement codes are shared or aggregated via DRGs. The EHR may include details about the administered product in a descriptive field. We also suggest mandatory use of NDCs and amendment of policy standards to capture manufacturer names and lot numbers in claims data.

\section{Other Considerations for Improving Biologic Pharmacovigilance}

With the increasing use of two- and three-dimensional barcoding, both at the bedside currently and potentially in the future as part of the implementation of the DQSA/ DSCSA, an opportunity for improving product traceability exists [8]. For patients who are administered a biologic, the information currently conveyed via barcodes is typically linked to information readily available at an institution's pharmacy; these data are only as rich as the information available for transfer into a barcoding system. As such, manual entry of long codes, batch numbers, manufacturer names, and other information (e.g., product expiration dates) currently prevents consistent availability and inclusion of this information in barcodes and $\mathrm{AE}$ reports; development of barcode product identifiers at the manufacturer level should standardize this type of information to allow greater ease of AE reporting. The DQSA/DSCSA are US federal mandates that require tracking of product information between trade partners [8]. Federal law currently does not require use of any particular technology for product tracing and allows paper-based records until 2017, at which point electronic records will be required by manufacturers; repackagers will be required to include electronic records in 2018. Wholesalers and dispensers will be required to only trade products with electronic identifiers beginning in 2019 and 2020, respectively. However, exemptions to this law may allow continued use of paper- 
Table 2 Drug Quality and Security Act/Drug Supply Chain Security Act (DQSA/DSCSA) gaps and proposed solutions

\begin{tabular}{ll}
\hline Barrier to patient-level biologic traceability & Method to overcome barrier \\
\hline $\begin{array}{l}\text { No requirement for electronic format of information to support } \\
\text { product tracing } \\
\text { No requirement for information to be available at the dose level }\end{array}$ & Universal barcode system used among trade partners \\
No requirement for transfer of data into patients' medical records & $\begin{array}{c}\text { Requirement for barcodes to be provided on primary } \\
\text { dose packaging } \\
\text { Automatic transfer of barcode information into patients' } \\
\text { medical records }\end{array}$ \\
\hline
\end{tabular}

based product tracing between manufacturers and licensed healthcare providers [8]. Additionally, there is no mandate for information to be available at the dose level; current product information requirements apply only to secondary packaging. Lastly, there is no requirement for transfer of data elements into a patient's medical record [8]. These three DQSA/DSCSA gaps can be bridged in the following ways: (1) promotion of barcode technology (e.g., GS1) as the major method of conveying information between partners; (2) requirements for barcodes on primary dose packaging; and (3) adoption of standards that require extension of rich product identifier information, conveyed as part of the federal law, past the final custodian of a biologic and into a patient's medical record (see Table 2).

\section{Conclusions}

Multiple product-specific identifiers should be available to reporters, who should be encouraged to include one or more such identifiers in spontaneous safety reports. The brand name can be one such identifier, but it is not required for prescribing or medical benefit-related claims-based record keeping. Also, because of the data structures of commonly used EHRs, NDCs and lot numbers cannot be relied on as redundant identifiers for reporters. The European solution of identifying products by a "trade name" comprising the INN and the manufacturer name (e.g., filgrastim Hexal) in the absence of a brand name will not work in the USA, because a concatenation of the nonproprietary name and the manufacturer name is not a recognized trade name, and the separate manufacturer name field is not commonly captured in health records or FAERS. Distinguishable nonproprietary names provide another retrievable identifier. The nonproprietary name is always available in health records when a brand name is not available or is not used, and this information could therefore be retrieved by a reporter for inclusion in a spontaneous report. As biosimilars are integrated into the existing pharmacovigilance systems, establishment of distinguishable nonproprietary names for all biologics is recommended to improve their traceability [36].
The biggest challenge for SRS and AS of biologics with multiple manufacturers lies in the physician office and hospital settings. Furthermore, substitution of products from multiple manufacturers without accurate documentation to distinguish between products will lead to inaccurate medication histories. Distinct HCPCS codes serve not only to implement Biologics Price Competition and Innovation Act provisions for Part B reimbursement but also to support application of the FDA Sentinel Initiative to future biologics with multiple manufacturers. The use of distinguishable nonproprietary names could also improve traceability in these medical and hospital benefit settings, where product identification is often homogenized to a shared nonproprietary name.

In the long term, technology systems must advance to ease the transfer of essential product identifier information into patient medical records and allow for interoperable access to this information by patients, physicians, and pharmacists. Although several gaps remain, the changes associated with implementation of the DQSA over the next decade provide an opportunity to take advantage of these technologies.

Acknowledgments The authors acknowledge Patrick Little, PhD, James Balwit, MS, and Miranda Tradewell, $\mathrm{PhD}$, whose work was funded by Amgen Inc., for assistance in writing this manuscript.

Author contributions GG and TF both contributed to writing this manuscript.

\section{Compliance with Ethical Standards}

Funding Funded by Amgen Inc.

Disclosures GG and TF are employees of and own stock in Amgen Inc. GG and TF have provided expert testimony on behalf of Amgen Inc. in support of legislation in US states that allows for automatic substitution of FDA-approved interchangeable biologic products with provisions to communicate and record biologic products dispensed.

Open Access This article is distributed under the terms of the Creative Commons Attribution-NonCommercial 4.0 International License (http://creativecommons.org/licenses/by-nc/4.0/), which permits any noncommercial use, distribution, and reproduction in any medium, provided you give appropriate credit to the original 
author(s) and the source, provide a link to the Creative Commons license, and indicate if changes were made.

\section{References}

1. US Food and Drug Administration. FDA approves first biosimilar product Zarxio. US Food and Drug Administration. 2015. http:// www.fda.gov/NewsEvents/Newsroom/PressAnnouncements/ ucm436648.htm. Accessed 1 July 2015.

2. US Food and Drug Administration. Guidance for industry: scientific considerations in demonstrating biosimilarity to a reference product. Rockville: US Food and Drug Administration; 2015.

3. Felix T, Johansson TT, Colliatie JA, Goldberg MR, Fox AR. Biologic product identification and US pharmacovigilance in the biosimilars era. Nat Biotechnol. 2014;32(2):128-30.

4. Casadevall N, Felix T, Strober BE, Warnock DG. Similar names for similar biologics. BioDrugs. 2014;28(5):439-44.

5. Casadevall N, Edwards IR, Felix T, Graze PR, Litten JB, Strober BE, et al. Pharmacovigilance and biosimilars: considerations, needs, and challenges. Expert Opin Biol Ther. 2013;13(7): 1039-47.

6. Zuniga L, Calvo B. Biosimilars: pharmacovigilance and risk management. Pharmacoepidemiol Drug Saf. 2010;19(7):661-9.

7. Kozlowski S, Woodcock J, Midthun K, Sherman RB. Developing the nation's biosimilars program. N Engl J Med. 2011;365(5): 385-8.

8. Drug Quality and Security Act, Public Law 113-54. 2013. http:// www.gpo.gov/fdsys/pkg/PLAW-113publ54/content-detail.html. Accessed 6 July 2015

9. Haerian K, Varn D, Vaidya S, Ena L, Chase HS, Friedman C. Detection of pharmacovigilance-related adverse events using electronic health records and automated methods. Clin Pharmacol Ther. 2012;92(2):228-34.

10. US Food and Drug Administration. Guidance for industry: E2E pharmacovigilance planning. US Food and Drug Administration. 2005. http://www.fda.gov/downloads/Drugs/GuidanceCompliance RegulatoryInformation/Guidance/ucm073107.pdf. Accessed 27 Oct 2014.

11. US Food and Drug Administration. How consumers can report an adverse event or serious problem to the FDA. US Food and Drug Administration. 2014. http://www.fda.gov/Safety/MedWatch/ HowToReport/ucm053074.htm. Accessed 27 Oct 2014.

12. Center for Drug Evaluation and Research, Center for Biologics Evaluation and Research. Guidance for industry: good pharmacovigilance practices and pharmacoepidemiologic assessment. Rockville: US Food and Drug Administration; 2005.

13. Pfizer. What is a safety signal? Pfizer. 2011. http://www.pfizer. com/files/health/medicine_safety/2-4_What_is_a_Safety_Signal. pdf. Accessed 9 Mar 2015.

14. Robinson S, Pool R, Giffin R. Emerging safety science: workshop summary. Washington, DC: National Academies Press; 2008.

15. Platt R, Madre L, Reynolds R, Tilson H. Active drug safety surveillance: a tool to improve public health. Pharmacoepidemiol Drug Saf. 2008;17(12):1175-82.

16. Vermeer NS, Straus SM, Mantel-Teeuwisse AK, Domergue F, Egberts TC, Leufkens HG, et al. Traceability of biopharmaceuticals in spontaneous reporting systems: a cross-sectional study in the FDA Adverse Event Reporting System (FAERS) and EudraVigilance databases. Drug Saf. 2013;36(8):617-25.

17. US Food and Drug Administration. Guidance for industry: postmarketing safety reporting for human drug and biological products including vaccines. US Food and Drug Administration. 2001. http://www.fda.gov/downloads/BiologicsBloodVaccines/
GuidanceComplianceRegulatoryInformation/Guidances/Vaccines/ ucm092257.pdf. Accessed 27 Oct 2014.

18. US Food and Drug Administration. CDER MAPP 5240.8: Handling of adverse experience reports and other generic drug postmarketing reports. US Food and Drug Administration. 2005. http://www.fda.gov/downloads/AboutFDA/CentersOffices/ OfficeofMedicalProductsandTobacco/CDER/ManualofPolicies Procedures/UCM079791.pdf. Accessed 27 Oct 2014.

19. US Food and Drug Administration. Quarterly data extract from the Adverse Event Reporting System (AERS). US Food and Drug Administration. 2011. http://www.docstoc.com/docs/159969837/ FDA-Asc-nts-database-description-ument. Accessed 27 Oct 2014.

20. Casadevall N. Immune-response and adverse reactions: PRCA case example. European Medicines Agency. 2009. http://www. ema.europa.eu/docs/en_GB/document_library/Presentation/2009/ 11/WC500011064.pdf. Accessed 26 Feb 2015.

21. Purcell RT, Lockey RF. Immunologic responses to therapeutic biologic agents. J Investig Allergol Clin Immunol. 2008;18(5):335-42.

22. Lietzan EF, Sim LE, Alexander EA. Biosimilar naming: how do adverse event reporting data support the need for distinct nonproprietary names for biosimilars? Food Drug Policy Forum. 2013;3(6):1-24.

23. Government Accountability Office. Food and Drug Administration response to heparin contamination helped protect public health; controls that were needed for working with external entities were recently added. Washington, DC: Government Accountability Office; 2010.

24. US Food and Drug Administration. Information on adverse event reports and heparin. US Food and Drug Administration. 2009. http://www.fda.gov/Drugs/DrugSafety/PostmarketDrugSafety InformationforPatientsandProviders/UCM112669. Accessed 29 Sept 2015.

25. Wetterhall SF, Noji EK. Surveillance and epidemiology. In: Noji EK, editor. The public health consequences of disasters. New York: Oxford University Press; 1997. p. 37-64.

26. Behrman RE, Benner JS, Brown JS, McClellan M, Woodcock J, Platt R. Developing the Sentinel system - a national resource for evidence development. N Engl J Med. 2011;364(6):498-9.

27. Schneider G, Kachroo S, Jones N, Crean S, Rotella P, Avetisyan $\mathrm{R}$, et al. A systematic review of validated methods for identifying anaphylaxis, including anaphylactic shock and angioneurotic edema, using administrative and claims data. Pharmacoepidemiol Drug Saf. 2012;21(s1):240-7.

28. Bohlke K, Davis RL, DeStefano F, Marcy SM, Braun MM, Thompson RS. Epidemiology of anaphylaxis among children and adolescents enrolled in a health maintenance organization. J Allergy Clin Immunol. 2004;113(3):536-42.

29. Huang F, Chawla K, Jarvinen KM, Nowak-Weegrzyn A. Anaphylaxis in a New York City pediatric emergency department: triggers, treatments, and outcomes. J Allergy Clin Immunol. 2012;129(1):162-8.

30. Johannes CB, Ziyadeh N, Seeger JD, Tucker E, Reiter C, Faich G. Incidence of allergic reactions associated with antibacterial use in a large, managed care organisation. Drug Saf. 2007;30(8):705-13.

31. Olson K. Alliance for Safe Biologic Medicines-prescriber survey. Alliance for Safe Biologic Medicines. 2012. http:// safebiologics.org/resources/wp-content/uploads/2012/09/ASBMSurvey-2.pdf. Accessed 27 Oct 2014.

32. Stergiopoulos S, Brown CA, Grampp G, Felix T, Getz KA. Identifying and quantifying the accuracy of product name attribution of US-sourced adverse event reports in MedWatch of somatropins and insulins. Ther Innov Regul Sci. 2015;9(5): 706-16. 
33. Directive 2010/84/EU of the European Parliament and of the Council of 15 December 2010 amending, as regards pharmacovigilance, Directive 2001/83/EC on the Community code relating to medicinal products for human use, L 348/74 (2010). Official Journal of the European Union. 2010. http://eur-lex. europa.eu/LexUriServ/LexUriServ.do?uri=OJ:L:2010:348:0074: 0099:EN:PDF. Accessed 31 Aug 2015.

34. Amgen Inc. Docket nos. FDA-2013-P-1153; FDA-2013-P-1398, respectively, non-proprietary naming of biosimilars. 2015. http:// www.regulations.gov/\#!documentDetail;D=FDA-2013-P-13980022. Accessed 10 Sept 2015.

35. Steinman MA, Chren MM, Landefeld CS. What's in a name? Use of brand versus generic drug names in United States outpatient practice. J Intern Med. 2007;22(5):645-8.

36. US Food and Drug Administration. Guidance for industry: nonproprietary naming of biological products. Rockville, MD. 2015.

37. World Health Organization. Biological qualifier: an INN proposal. World Health Organization. 2014. http://www.who.int/ medicines/services/inn/bq_innproposal201407.pdf?ua=1. Accessed 27 Oct 2014.

38. US Food and Drug Administration. Code of Federal Regulations. 21CFR § 201.2 Drugs and devices; National Drug Code numbers. US Food and Drug Administration. 2012. http://www.accessdata. fda.gov/scripts/cdrh/cfdocs/cfCFR/CFRSearch.cfm?fr=201.2. Accessed 29 Sept 2015.

39. US Food and Drug Administration. Instructions for completing form FDA 3500. US Food and Drug Administration. 2013. http:// www.fda.gov/Safety/MedWatch/HowToReport/DownloadForms/ ucm149236.htm. Accessed 8 July 2014.

40. US Food and Drug Administration. Form FDA 3500. US Food and Drug Administration. 2013. http://www.fda.gov/downloads/ AboutFDA/ReportsManualsForms/Forms/UCM163919.pdf. Accessed 5 Aug 2014.

41. Centers for Disease Control and Prevention, US Food and Drug Administration. Vaccine Adverse Event Reporting System. US Food and Drug Administration. 2014. https://vaers.hhs.gov/index. Accessed 1 July 2015.

42. Goldman GS, Miller NZ. Relative trends in hospitalizations and mortality among infants by the number of vaccine doses and age, based on the Vaccine Adverse Event Reporting System (VAERS), 1990-2010. Hum Exp Toxicol. 2012;31(10):1012-21.

43. National Institute of Standards and Technology. NIST NCPDP initial assessment: standards compatability in medication reconciliation. National Institute of Standards and Technology. 2011. http://healthcare.nist.gov/resources/docs/FirstAmerican/NIST_ NCPDPStandardsCompatibilityMedRec.pdf. Accessed 6 Aug 2014.

44. Hennessy S, Leonard CE, Platt R. Assessing the safety and comparative effectiveness of follow-on biologics (biosimilars) in the United States. Clin Pharmacol Ther. 2010;87(2):157-9.
45. Getz KA, Stergiopoulos S, Kaitin KI. Evaluating the completeness and accuracy of MedWatch data. Am J Ther. 2014;21(6):442-6.

46. DiMartino LD, Curtis LH, Williams RL, Abernethy DR, Schulman KA. Using Medicare administrative data to conduct postmarketing surveillance on follow-on biologics: issues and opportunities. Food Drug Law J. 2008;63:891-900.

47. Grampp G, Bonafede M, Felix T, Li E, Malecki M, Sprafka JM. Active and passive surveillance of enoxaparin generics: a case study relevant to biosimilars. Expert Opin Drug Saf. 2015;14(3):349-60.

48. Centers for Medicare and Medicaid Services. Medicare program: hospital outpatient prospective payment and ambulatory surgical center payment systems and quality reporting programs; short inpatient hospital stays; transition for certain medicare-dependent, small rural hospitals under the hospital inpatient prospective payment system. Centers for Medicare and Medicaid Services. 2015. https://www.federalregister.gov/articles/2015/07/08/201516577/medicare-program-hospital-outpatient-prospective-pay ment-and-ambulatory-surgical-center-payment. Accessed 16 July 2015.

49. Yesner DL. Where will biosimilars fit in federal drug pricing programs. In: Pharmaceutical Law \& Industry Report. Morgan Lewis \& Bockius LLP. 2011. http://www.morganlewis.com/ pubs/where-will-biosimilars-fit-in-federal-drug-pricing-programsbnas-ipharmaceutical-law-industry-reporti. Accessed 24 Sept 2014.

50. Social Security Administration. Section 1847A: use of average sales price payment methodologies. Social Security Administration. http://www.ssa.gov/OP_Home/ssact/title18/1847A.htm. Accessed 8 Sept 2014.

51. US Food and Drug Administration. Guidance for industry: biosimilars: questions and answers regarding implementation of the Biologics Price Competition and Innovation Act of 2009. Rockville: US Food and Drug Administration; 2015.

52. State of Delaware. State Bill 118. An act to amend Title 24 of the Delaware code relating to pharmacists. State of Delaware. 2014. http://legis.delaware.gov/LIS/lis147.nsf/vwLegislation/SS+1+ FOR+SB+118/\$file/legis.html?open. Accessed 1 July 2015.

53. State of Massachusetts. Bill H.3734: an act relative to the substitution of interchangeable biosimilars. State of Massachusetts. 2014. https://malegislature.gov/Bills/188/House/H3734/History. Accessed 5 Aug 2014.

54. Federal Register, Vol 77, No. 95, 2012. http://www.gpo.gov/ fdsys/pkg/FR-2012-05-16/pdf/2012-11543.pdf. Accessed 8 Sept 2014.

55. Platt R, Carnahan RM, Brown JS, Chrischilles E, Curtis LH, Hennessy S, et al. The US Food and Drug Administration's MiniSentinel program: status and direction. Pharmacoepidemiol Drug Saf. 2012;21(S1):S1-8. 